\title{
Astro Particle Physics from Space
}

Roberto Battiston ${ }^{1}$

Dipartimento di Fisica and Sezione INFN, I-06121 Perugia Italia

Abstract. We review how some open issues on Astro Particle physics can be studied by space born experiments in a complementary way to what is being done at underground and accelerators facilities

\section{At the beginning it was Astro Particle}

This figure shows the conference photo taken at the 1939 Chicago Symposium on Cosmic Rays. Among the partecipants we find a quite exceptional group of physicists: Kohloster, Bethe, Shapiro, Compton, Teller, Eckart, Gouldsmit, Anderson, Oppenheimer, Hess, Wilson, Rossi, Auger, Heisenberg, Weehler and

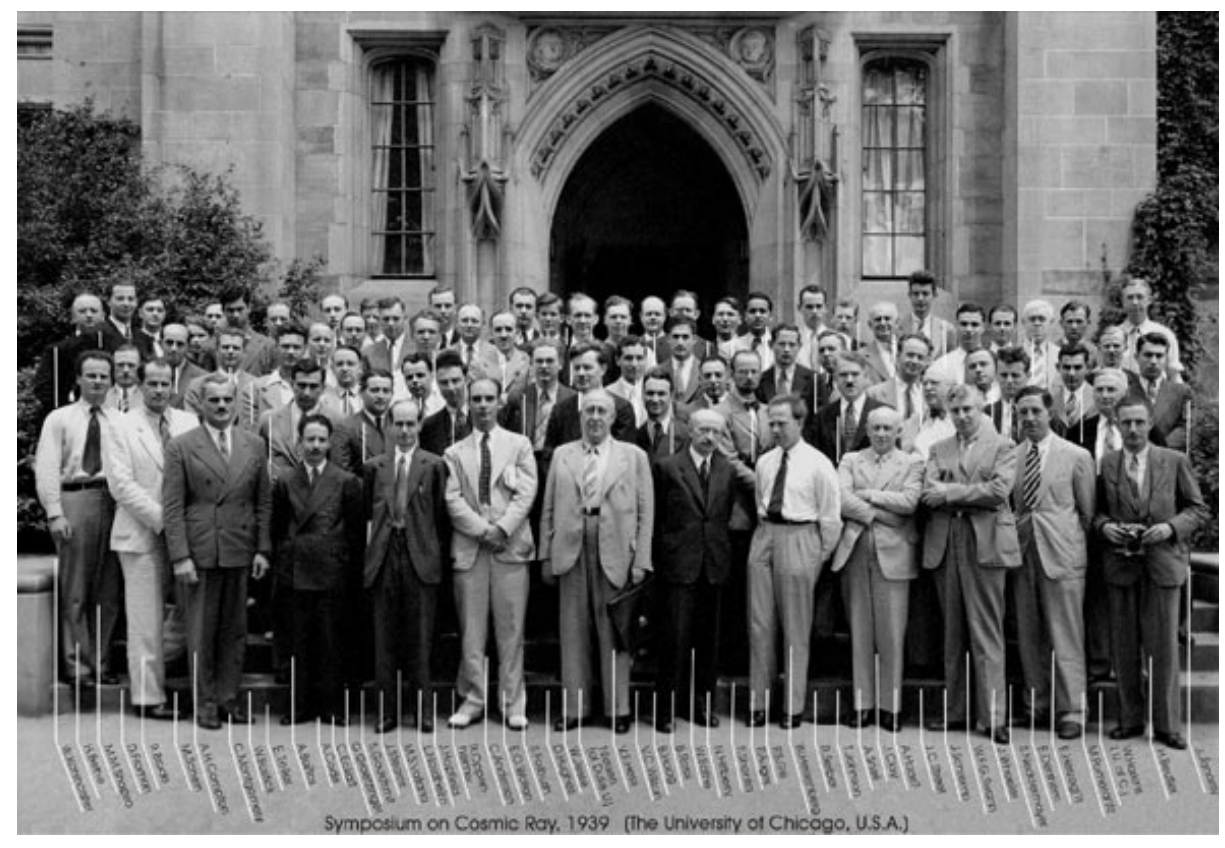

1

${ }^{1}$ Invited Talk at the ESO-CERN-ESA Symposium on Astronomy, Cosmology and Fundamental Physics, March 4-7 2002, Garching, Germany 
many other which are the among the fathers of the modern physics, based on Quantum Mechanics, Elementary Particles and Fundamental Forces. Why Cosmic Rays, discovered by Hess nearly 30 years before were, still in 1939, such an interesting topics for these distinguished scientists?

The answer lies in Table 1. In the years preceding 1937 both the first antiparticle (the positron) and the first unstable elementary particles $\left(\mu^{ \pm}\right)$were discovered in Cosmic Rays. Many more particles were to be discovered during the following years analyzing the Cosmic Radiation, making Cosmic Rays symposia very exciting until at least 1953, when experiments at accelerators started to systematically discover new elementary particles while Cosmic Rays experiments suddenly stopped finding them.

Table 1. Discovery of elementary particles

\begin{tabular}{llll}
\hline Particle & Year & Discoverer (Nobel Prize) & Method \\
\hline$e^{-}$ & 1897 & Thomson (1906) & Discharges in gases \\
$p$ & 1919 & Rutherford & Natural radioactivity \\
$n$ & 1932 & Chadwik (1935) & Natural radioactivity \\
$e^{+}$ & 1933 & Anderson (1936) & Cosmic Rays \\
$\mu^{ \pm}$ & 1937 & Neddermeyer, Anderson & Cosmic Rays \\
$\pi^{ \pm}$ & 1947 & Powell (1950), Occhialini & Cosmic Rays \\
$K^{ \pm}$ & 1949 & Powell (1950) & Cosmic Rays \\
$\pi^{0}$ & 1949 & Bjorklund & Accelerator \\
$K^{0}$ & 1951 & Armenteros & Cosmic Rays \\
$\Lambda^{0}$ & 1951 & Armenteros & Cosmic Rays \\
$\Delta$ & 1932 & Anderson & Cosmic Rays \\
$\Xi^{-}$ & 1932 & Armenteros & Cosmic Rays \\
$\Sigma^{ \pm}$ & 1953 & Bonetti & Cosmic Rays \\
$p^{-}$ & 1955 & Chamberlain, Segre' (1959) & Accelerators \\
anything else & $1955 \Longrightarrow$ today & various groups & Accelerators \\
$m_{\nu} \neq 0$ & 2000 & KAMIOKANDE & Cosmic rays \\
\hline
\end{tabular}

If Cosmic Rays have been instrumental to give birth to particle physics during the first half of the past century, starting from the fifties, however, accelerators have been the tools for the experimental triumph of the Standard Model of Particle Physics, including the discovery of the electro-weak bosons at CERN or of the heavy sixth quark at Fermilab. 
During the last ten years, however, the rate of discoveries at accelerators seems significantly reduced, possibly because of the limited energy scale which can be tested at existing or future facilities. A growing number of physicists is then turning again to $\mathrm{CR}$ with new experimental techniques aiming to extend by orders of magnitude the sensitivities reached by past experiments.

In particular a number of space born experiments have been proposed to measure, with unrivalled accuracy, the composition of primary high energy CR, searching for new phenomena not accessible to present accelerators.

In this paper we will review these experiments and their physics potential. The paper is organized in two parts. In the first part I will discuss the charac-

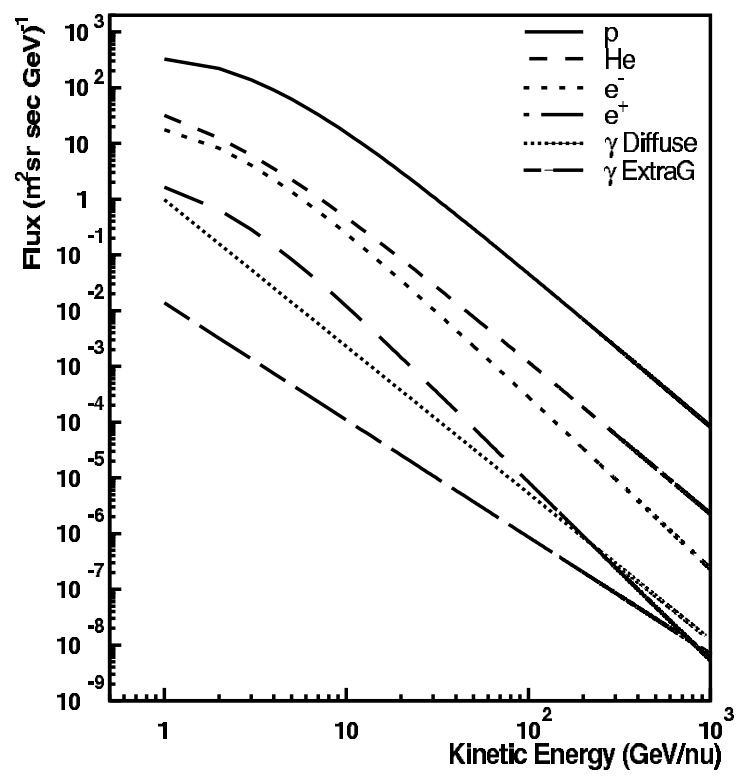

Fig. 1. High Energy Cosmic Rays composition [1]

teristics of the CR flux, the beam nature gives us, reviewing the status of our knowledge of their energy spectrum and composition. In the second part I will discuss some of the space born experiments planned in the next future which will contribute to the quest for answers to various unsolved questions in Astro Particle physics.

\section{Understanding Nature's Beam}

The Universe communicate with us by sending to Earth a continuous flow of radiation of different kind. Here we are interested to the high energy part of the spectrum $(E>O(G e V))$. Cosmic Rays traditionally refer to the charged 
component of high the energy particles travelling through the galaxy (Figure $1)$ : in order of abundance $p \sim 80 \%,{ }^{4} \mathrm{He} \sim 15 \%, e^{-}: O(1 \%), e^{+}: O(0.1 \%), \bar{p}:$ $O(0.01 \%))$. In addition to $p$ and ${ }^{4} H e$, there is a composite hadronic component including all other nuclei and long lived isotopes, totalling about a few $\%$ of the total CR flux.

There are however two other forms of energetic radiation which are relevant for Astro Particle physics. The first is the high energy part of the electromagnetic spectrum, gamma rays above $\sim 100 \mathrm{MeV}$. Their flux is at the level of $10^{-5}$ of the CR flux. Gamma rays have an important property that charged CR do not have, namely they travel along straight lines, undisturbed by the magnetic field and reproducing the images of their sources.
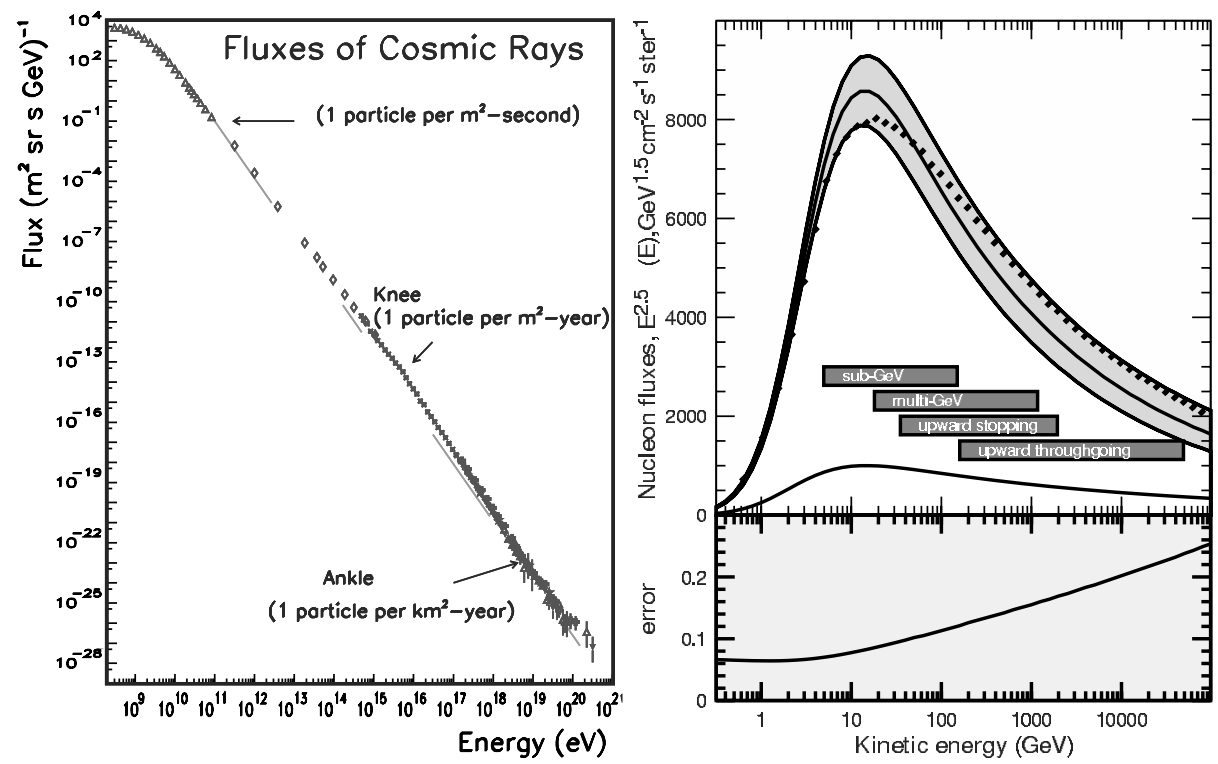

Fig. 2. Left: the flux of charged cosmic rays. Right: measurement and uncertainty on the primary $\mathrm{CR}$ spectrum (proton and He).The accurate knowledge of this part of the CR spectrum is important for the understanding of the atmospheric neutrino flux [2].

The second component are the neutrinos, which would also allow source imaging: at high energy the primary neutrino flux is much lower and steeper with increasing energy than for charged CR, with the exception of the secondary atmospheric component induced by high energy CR hitting the atmosphere. Around $1 \mathrm{GeV}$ the neutrino flux is of the order of the flux of high energy gamma rays, but because of their low cross section, $\nu^{\prime} s$ are much harder to detect.

If we are plan to use this energetic radiation to search for new particles or new effects, we must know the properties of Nature's beam with the best possible 
accuracy. Which is our current level on the knowledge of the Cosmic Radiation and what we can expect in the coming future?

\section{The charged CR component}

\subsection{Charged hadrons}

Over the last 40 years the hadronic CR component has been measured systematically, using balloons (mostly), space born (sometimes), and ground based detectors. The most complete information is obtained by particle spectrometers, experiments able to measure directly the CR composition through the determination of the charge, the sign of the charge, the momentum and the velocity of each particle. Often, however, simpler apparatus were used, based on calorimeters, emulsion chambers or Cerenkov detectors: in these cases only partial information were obtained. Until recently the most sophisticated CR spectrometers were flown on balloons operating between 30 and $35 \mathrm{~km}$ of height (BESS[3], MASS[4], CAPRICE[5], IMAX[6]) but in 1998 a large magnetic spectrometer, AMS[7], has been operated in space, providing for the first time a very precise measurement on the composition of primary $\mathrm{CR}$ before their entrance in the atmosphere.

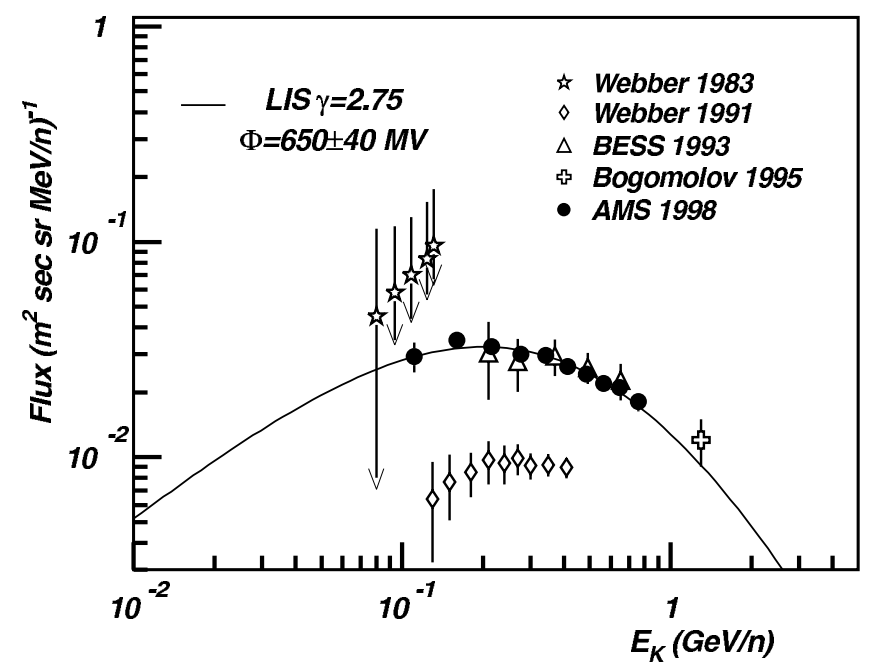

Fig. 3. Cosmic deuteron measurements[8-12]

The flux of the main CR component, the protons, is known with $5-10 \%$ accuracy up to $\sim 200 \mathrm{GeV}$, and with $10-30 \%$ accuracy up to $\sim 100 \mathrm{TeV}$ (Figure 2) [2]. Helium flux is known with $10 \%$ accuracy up to $\sim 10 \mathrm{GeV}$ but above this energy the measurements are rather poor. Light $Z>2$ nuclei have been 
measured with about $5 \%$ accuracy only up to $\sim 35 \mathrm{GeV}$. It would be important to extend the energy range for precise measurements of hadrons up to about $10 \mathrm{TeV}$ because they are the source of the atmospheric neutrinos used for the determination measurement of the neutrino mass in underground experiments.

For the study of CR composition, precise measurements of some stable isotopes like Deuterium, Boron and Carbon or long-lived isotopes like ${ }^{9} \mathrm{Be}$ are particularly important to understand the propagation and trapping mechanisms of CR in our Galaxy. Accurate measurements of $D$ are available only up a few $G V$ of rigidity (Figure 3 ) while the knowledge of the cosmologically important ratio ${ }^{9} \mathrm{Be} /{ }^{10} \mathrm{Be}$ is very poorly known above about hundred $M V$ of rigidity.

\subsection{Antiprotons}

Antiprotons are a rare but interesting hadronic component of high energy CR, because they could be produced by exotic sources like antimatter dominated regions or by the decay or annihilation of new particles. Their flux ratio to protons is at the level of $O\left(10^{-4}\right)$ at kinetic energies around $1 \mathrm{GeV}$. This rate is in agreement with the expectation that $\bar{p}$ are produced in high energy CR interactions with the interstellar medium. However in more than 40 years of experiments with balloons, only a few thousands $\bar{p}$ have been measured, mostly with energies below $10 \mathrm{GeV}$ (Figure 4). Statistical errors are then quite large, in particular below $1 \mathrm{GeV}$ and above $10 \mathrm{GeV}$ : also systematic errors due to uncertainties in the modelling of the propagation in the Interstellar Medium or of the solar modulation are still at the level of $20-30 \%$.

\subsection{The charged leptons}

Due to their lower flux $\left(\sim 0.5 \%\right.$ for $e^{-}$and $\sim 0.05 \%$ for $e^{+}$around $\left.1 \mathrm{GeV}\right)$ and their steeply falling spectrum, statistical uncertainties on the measurement of the spectra of the two stable leptons are larger; the experimental situation gets very confused above a few $\mathrm{GeV}$ (Figure 5). Precision measurement of electrons and positrons are important since, being their fluxes quite low and with a strong charge asymmetry, contribution form exotic sources like supersymmetric particles annihilation could distort the $e^{+} / e^{-}$ratio at a level which could detectable by an high precision experiment[15].

\subsection{Future experiments}

During the next 5 years our knowledge of the flux and composition of CR will be greatly improved thanks to two space borne spectrometers, PAMELA on a Russian rocket in 2003-2006[16] and AMS-02 on the International Space Station on 2006-2009[17]. These two experiments will increase the statistical samples of charged CR by a few to several orders of magnitude. AMS-02, in particular, thanks to its strong magnetic field and large geometrical factor, will be able to extend these measurements well in to the $\mathrm{TeV}$ region, covering a region which is 


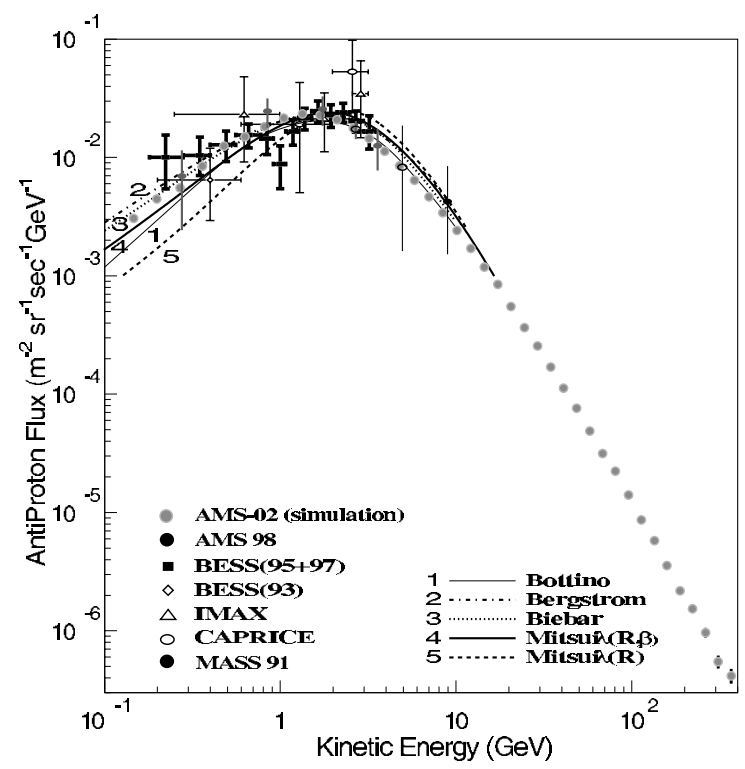

Fig. 4. Compilation of antiproton measurements. A simulated measurement of the AMS-02 experiment on the ISS is also reported. For all balloon data and models see[13] and reference therein. For AMS data see[7]

interesting for various physics topics and which today is very poorly known. For a discussion on the improvement expected from AMS-02 on the hadronic CR component see [18]. Space born experiments operating for three years or more are much more accurate that balloon flights lasting only about one day. Long duration balloons experiments lasting for 20 days or more, however, could be competitive, as it is shown in Table 2 .

Table 2. Future CR spectrometers

\begin{tabular}{lcccccc}
\hline & $\begin{array}{l}\text { Aperture } \\
\left(\mathrm{cm}^{2} \mathrm{sr}\right)\end{array}$ & $\begin{array}{l}\text { Duration } \\
(\text { days })\end{array}$ & $\begin{array}{l}\text { Altitude } \\
(\mathrm{km})\end{array}$ & $\begin{array}{l}\text { Latitude } \\
(\text { degrees })\end{array}$ & $\begin{array}{l}\text { Launch } \\
(\text { year })\end{array}$ & $\begin{array}{l}\text { Area*Time } \\
(\text { AMS-01 })\end{array}$ \\
\hline AMS-01 & 2300 & 10 & $320-390$ & $<51.7$ & 1998 & 1.0 \\
PAMELA & 21 & 1000 & 690 & 70 & 2003 & 1.1 \\
BESS Polar & 3000 & 20 & 36 & $>70$ & 2004 & 2.6 \\
AMS-02 & 5000 & 1000 & $320-390$ & $<51.7$ & 2006 & 217.0 \\
\hline
\end{tabular}

While at high energy the AMS-02 will be the most performing experiment, the Polar BESS[19] long duration flights, thanks to BESS large acceptance, will 

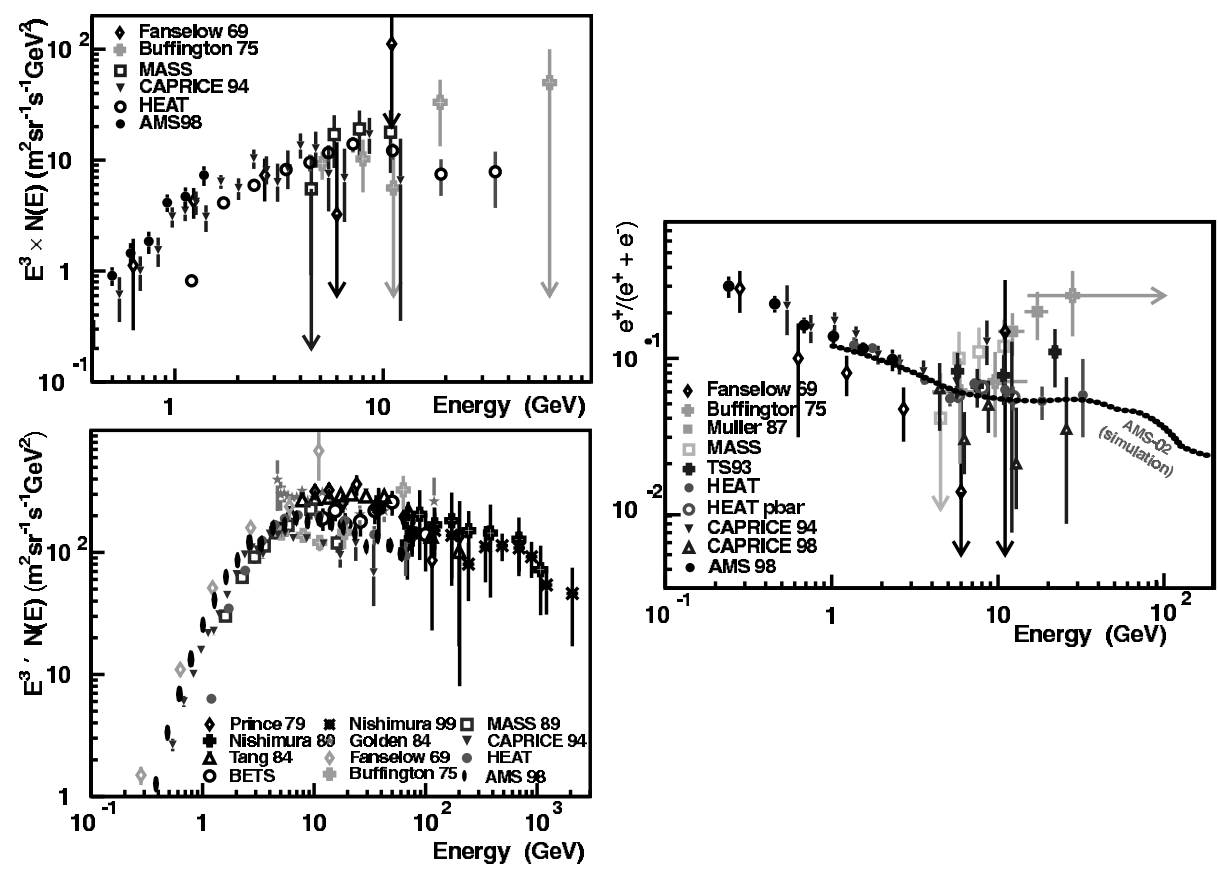

Fig. 5. Compilation of $e^{+}$(top left), $e^{-}$(bottom left) and $e^{+} /\left(e^{-}+e^{+}\right)$(right) data: labels corresponds to the various references cited in [14]. A simulated long exposure result from AMS-02 is also included, for the case $m_{\chi}=275 \mathrm{GeV} / \mathrm{c}^{2}[15]$. All experiments are balloon borne spectrometers with the exception of AMS 98 and AMS-02 which are space borne spectrometers

have a good sensitivity to the lower energy part of the CR spectrum and will perform a precise measurement of low energy $\bar{p}$. On the other side the space borne PAMELA spectrometer, in spite of its small aperture, is a timely experiment and will have a chance to perform high statistic $\mathrm{CR}$ measurements in the period between AMS-01 and AMS-02.

At energies around the knee $\left(\sim 10^{15} \mathrm{eV}=1 \mathrm{PeV}\right)$ and above, all measurements come nowadays from large area ground based arrays. At these energies the CR elemental composition can be determined only by modelling the interaction of $\mathrm{CR}$ with the atmosphere and it is affected by large uncertainties. To solve this problem a long duration, large acceptance, space borne experiment, ACCESS, has been considered[20] either for deployment on the International Space Station or as a free flyer.

Ground based large area arrays have measured the CR spectrum (not the composition) up to Extreme Energies $\left(10^{21} \mathrm{eV}=1 \mathrm{ZeV}\right)$ and they are at the moment the only experiments able to measure these very rare events. At these energies the flux is so low that eventually it will becomes unpractical to extend the surface or the time of exposure of ground based experiments. The largest 
experiment of this kind, Auger[21], is being built right now. The next step will, however, to look to the fluorescence induced by the EECR showers from space, as proposed by EUSO[22], an experiment planned on the International Space Station and capable to collect 10-50 times more statistics than Auger or by KLYPVE[23] planned on a Russian free flyer.

\section{The neutral CR component}

\subsection{High energy gamma rays}

High energy gamma rays are a rare $\left(O\left(10^{-5}\right)\right)$ component of the cosmic radiation which is not traditionally included in a $\mathrm{CR}$ review paper. However high energy gamma rays are produced by the same sources producing high energy CR and carry complementary information. They should then be considered when discussing Astro Particle physics, in particular since their study could give important contributions to the understanding the problem of the origin of dark matter. Their energy spectrum could, in fact, be influenced by exotic sources like neutralino annihilations taking place at the center of the galaxy. Most of the high energy gamma rays data have been collected by the EGRET experiment on the CGRO satellite during the $90^{\prime} s$. Since the end of the CGRO program $(1999-2000)$ there are no experiments measuring high energy gamma rays in space. During the present decade there will be three space borne experiments which will be able to measure high energy gamma rays: AGILE[24] (2003) a small scientific mission of the Italian Space Agency, AMS-02 on the International Space Station (2006)[25] and GLAST[26] (2007). These experiments will be able to cover the region up to $\sim 300 \mathrm{GeV}$ competing with ground base Cerenkov detectors which meanwhile will try to lower their threshold below $\sim 50 \mathrm{GeV}$.

\subsection{High energy neutrinos}

Neutrinos are a very important part of the Cosmic Radiation. Their spectrum (Figure 6) extend over several orders of magnitudes like for the charged CR component, but is rich of features coming from the various physical process at work.

Neutrinos have a two great advantages and one disadvantage with respect to charged CR:

Advantages:

- 1) they travel on straight lines so neutrino astronomy is a possibility;

- 2) they have a small interaction cross section so they are basically unaffected by the GZK[27] cutoff and can reach us from the edges of the universe (see Figure 7).

Disadvantage:

- l) they have a small interaction cross section so their detection is problematic, requiring very large volumes of matter. 


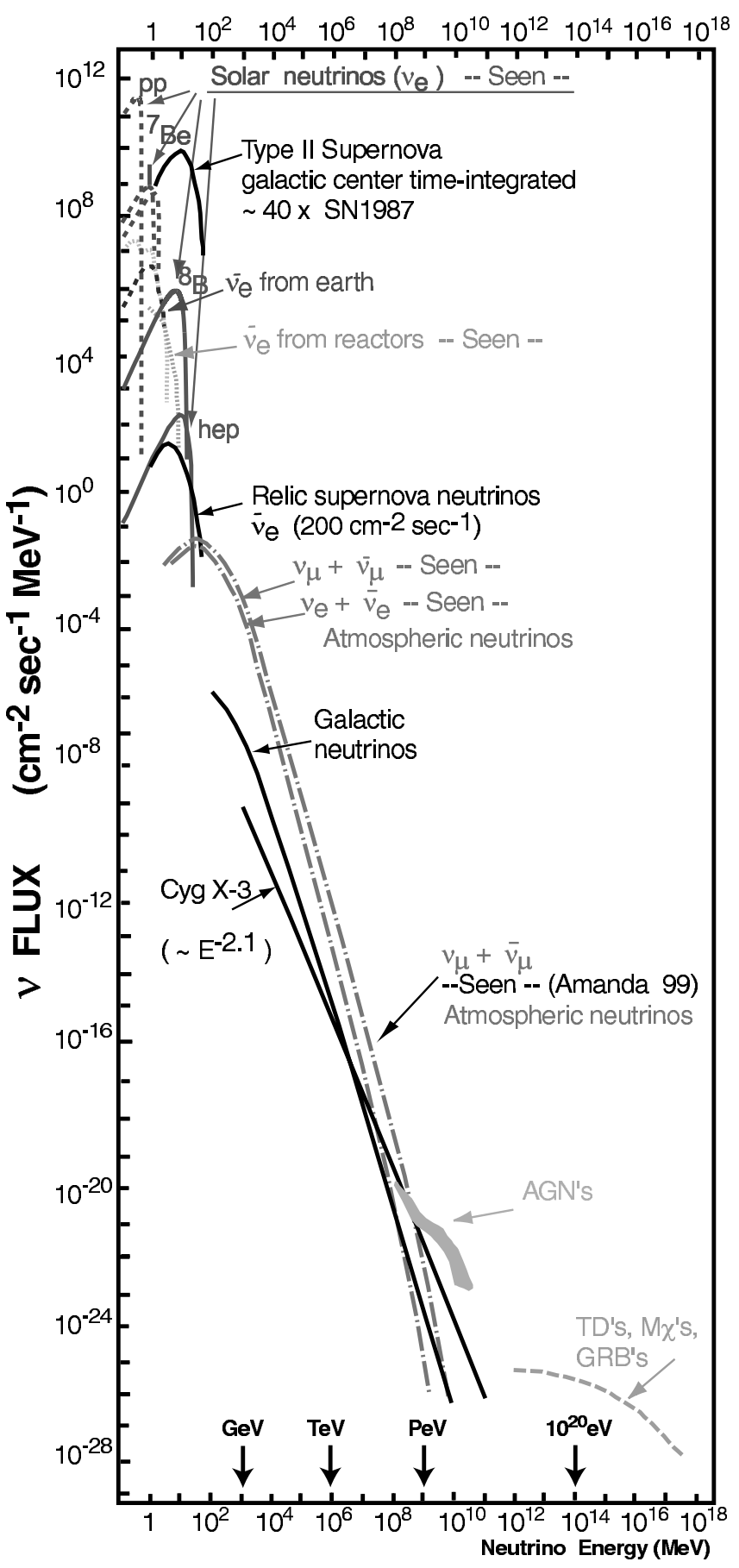

Fig. 6. The Cosmic neutrinos spectrum 
Many of the neutrinos spectral features have been measured ( $\nu$ from the sun, from $S N 1987$, from reactors, from the atmosphere), some may be on the verge of being seen as $\nu$ 's of galactic origin[28] while other are expected to exist but are still undetected (relic neutrinos, neutrinos from AGN's). Some could be produced by exotic sources, like superheavy particles or topological defects. The interest of neutrinos is their capability to escape the GZK cutoff up to extreme energies: they are the only particle that can reach us from the edges of the universe, carrying information about the status of space, time and matter, before the recombination took place (Figure 7).

The main difficulty for neutrino detection is related to the mass required for their detection. The only way to access energies of the order of $10^{14} \mathrm{eV}$ or above is to use the earth surface layers as targets, like the atmosphere, ice or water. In the case of the atmosphere the fluorescence light emitted by the developing shower could be detected from a space experiment able to monitor a sufficiently large area of our planet. This is the purpose or the EUSO[22] experiment on the International Space Station, expected to increase the sensitivity of the Auger array by more than an order of magnitude by the end of the decade.

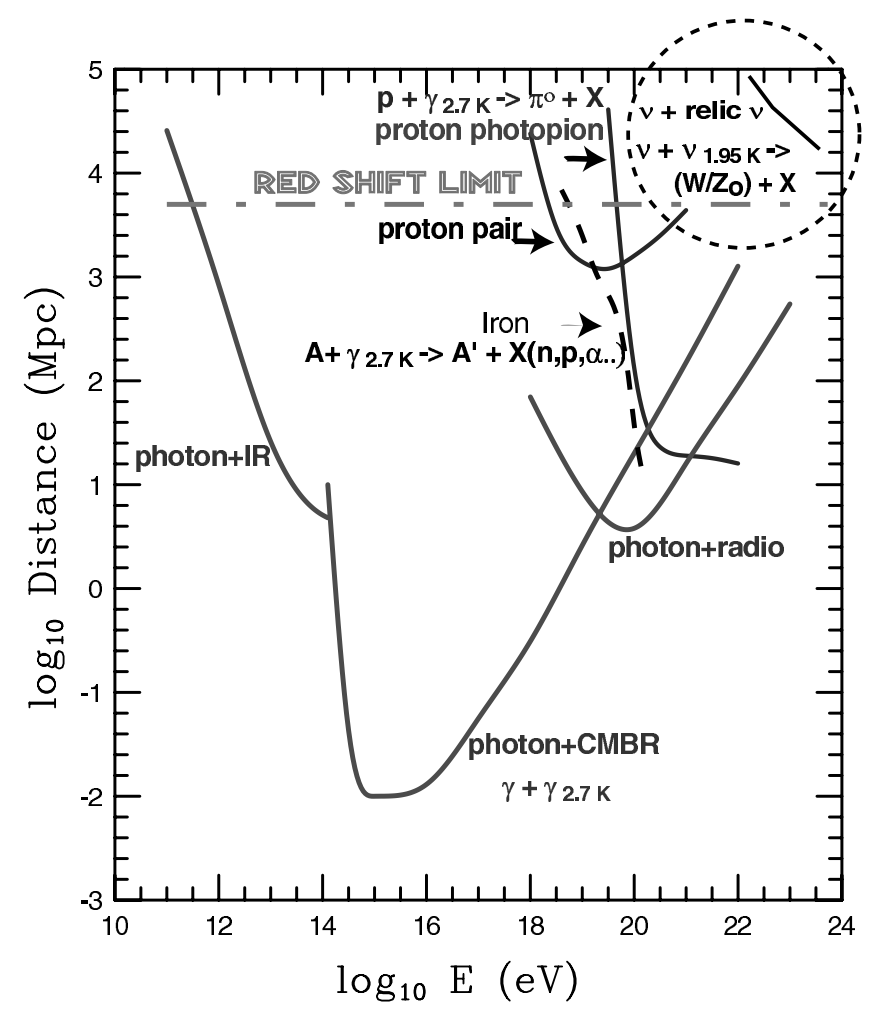

Fig. 7. Energy dependence of GZK cutoff for different CR species[29] 


\section{Searching for new particles}

In this section now briefly discuss the physics potential of the accurate study of the cosmic radiation. An area of clear interest for particle physics is obviously the search of new states of matter or for new particles.

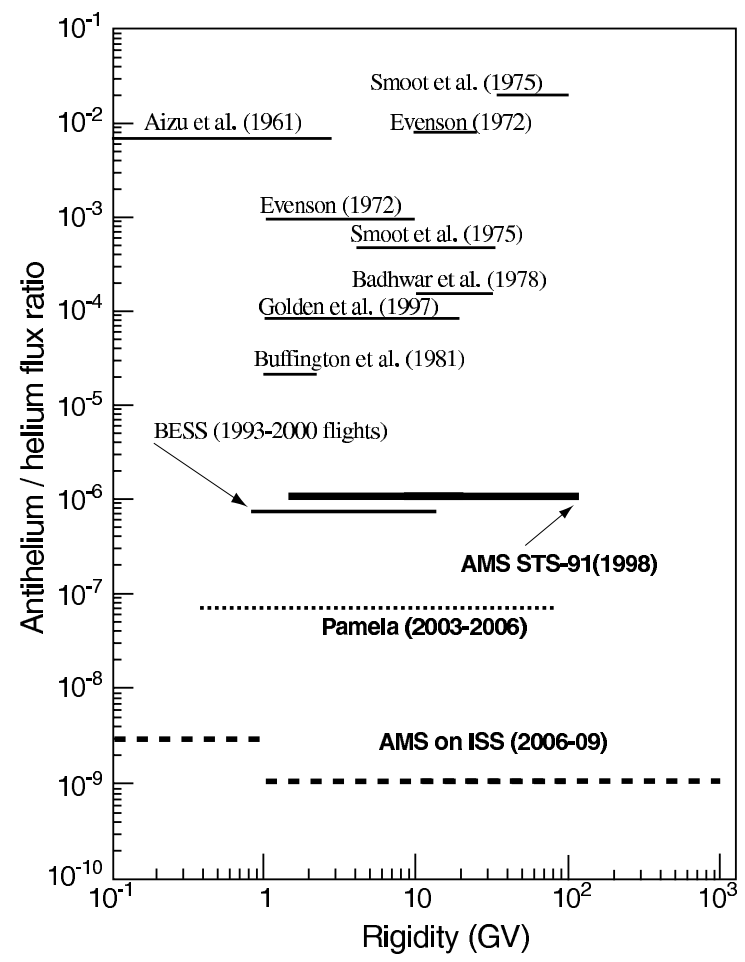

Fig. 8. Antimatter limits. For the references see [30]

\subsection{Direct search for nuclear anti-matter}

The disappearence of antimatter [31-33] is one of the most intriguing puzzles in our current understanding of the structure of the Universe. Absence of nuclear antimatter from the scale of our galaxy to the scale of the local supercluster is experimentally established at the level of 1 part in $10^{6}$ by direct CR searches and indirect methods like the study of the energy spectrum of the diffuse gamma ray flux. For a genuine antimatter signal one should look to nuclei heavier that $\bar{p}$ since secondary $\bar{p}$ can be easily produced at the level $10^{-4}$ in high energy hadronic interaction of CR with the IM. This probability quickly vanishes with increasing atomic number. For $\bar{D}$ the secondary production is at the level of $10^{-8}$ or less and already for ${ }^{4} \bar{H} e$ it is well below $10^{-12}$. 


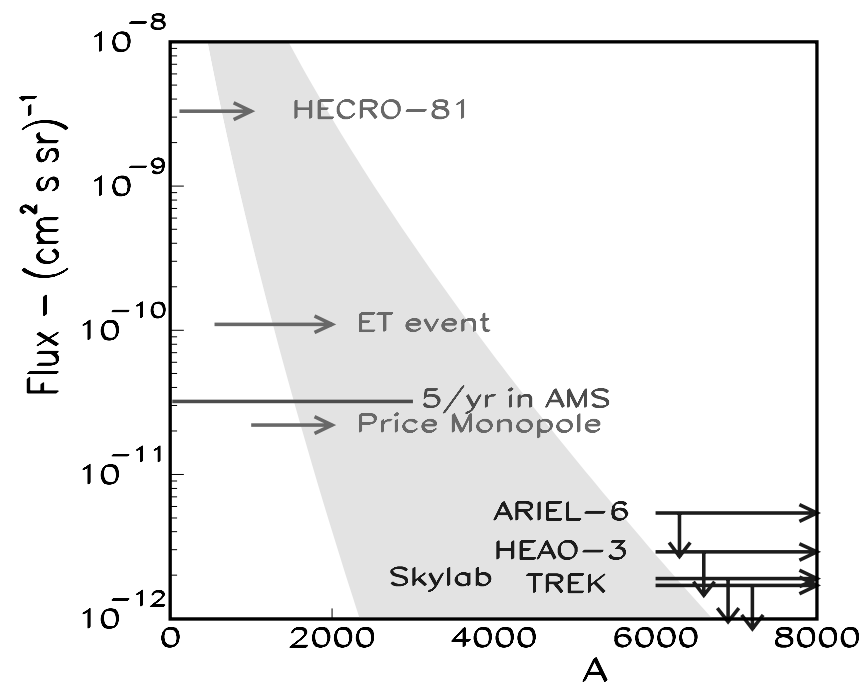

Fig. 9. Sensitivity to strangelets in AMS-02

This is why the unambiguous observation of a couple of ${ }^{4} \bar{H} e$ at the level of one part in a billion or more would have profound implications on our understanding of baryogenesis. During the last 35 years experiments on balloons have pushed the limit on the ${ }^{4} \bar{H} e$ at the level of less than about one part in a million (Figure 8). Recently the AMS-01 spectrometer, during a 10 days precursor flight on the Shuttle, has reached the same level of sensitivity. In the coming years PAMELA, first, and AMS-02, later, will eventually reach a sensitivity a thousand time better, reaching rigidities of the order of a TV.

\subsection{Direct search for strange states of matter}

Strangelets[34] are a stable state of nuclear matter containing a large fraction of strange quarks. Such states could have developed during the cosmological quark-hadron phase transition $10^{-5} s$ after the Big Bang or in the high density conditions of compact supernova remnants, which might then be strange stars composed of quark matter rather than neutron stars. These stars could send to space fragments of stable strange nuclear matter during catastrophic events like collisions with other strange stars. These fragment would have variable mass but a peculiar charge to mass ratio. For instance in the case of color-flavor locked strangelets we obtain a charge to mass relation of the type $Z=0.3 A^{2 / 3}$. These particles would look like high mass, low mass/charge ratio cosmic rays which could be easily identified in a space born magnetic spectrometer like AMS-02. Figure 9 shows the sensitivity to strangelets expected for AMS-02 after three years on the International Space Station[35]. 


\subsection{Indirect search for Dark Matter}

The presence at all scales in our universe of a non luminous components of matter, Dark Matter (DM) $[37,38]$, is possibly the most fascinating problem in Astro Particle physics. A viable solution, if not the most viable solution[36] to this problem, is given by the Lightest Supersymmetric Particle (LSP), the neutralino $(\chi)$. Supersymmetry links the existing Standard Model particles to a set of new, heavier, super particles through $R$-parity conservation, where $R$ is a combination of the particle spin, lepton and baryon numbers, $R=(-1)^{3 B-L+2 S}$. The conservation of $R$-parity requires that the LSP is stable. LEP results suggests that the LSP is heavy $\left(m_{\chi}>45 \mathrm{GeV}\right)[39]$ and then these particles can be a good DM candidate in the Cold Dark Matter (CDM) scenario.

Unfortunately SUSY is a theory with many parameters still poorly constrained. The LSP (neutralino) can be expressed as superposition of the neutral gauge $\left(g\right.$ and $W$ ) and Higgs boson superpartners $\left(H_{01}, H_{02}\right)$ :

$$
\chi=N_{11} B+N_{12} W_{3}+N_{13} H_{01}+N_{14} H_{02}
$$

The parameters of this superposition define the $\chi$ properties, like the mass, the annihilation cross section and branching ratios into detectable particles. These parameters can also be related to the $\chi$ cosmological density, which can be constrained in the interesting region $0.1<\Omega_{D M}<0.3$, in agreement with the recent astrophysical results[40].

$\chi$ annihilation would take place in the most dense regions of our galaxy, e.g. its center or in other existing DM clumps. These regions could be the source of prompt high energy CR without need of an acceleration mechanism. In order to detect these prompt $\mathrm{CR}$ we study rare CR components where the effects of the exotic $\chi$ contributions would be detectable against backgrounds due to the primary spectrum. Rare CR components like high energy $\bar{p}[37], e^{+}[15], \bar{D}[42]$ and $\gamma[43]$ have been suggested as potential indirect signatures for cold $D M$.

In Figure 5 we give an example of spectral distortions induced by $\chi$ annihilations on the $e^{+} /\left(e^{+}+e^{-}\right)$ratio. Quite interesting is the case of $\bar{D}$ production, since it has been suggested[42] that a $\bar{D}$ signal at kinetic energies below $\sim 1 \mathrm{GeV}$ would be a strong indication for $\chi$ annihilation. In the case of high energy $\gamma$ rays the spectral deformation due to $\chi$ annihilation is expected to have a strong spatial dependence, mimicking the DM halo structure which might have more than one clumps in our galaxy. For a subset of SUSY models this flux of HE $\gamma$ rays could be detectable by space born experiments (Figure 10).

For many SUSY models the indirect search for $\chi$ in CR will be a difficult undertaking: SUSY predictions depends in fact from several parameters and the limited precision in the knowledge of CR composition and spectra will challenge the unambiguous detection of a $\chi$ signal. Before the advent of the LHC, however, the physics potential of SUSY searches in space is promising[41,39]. In fact most of the cosmologically relevant SUSY parameter space can be explored by CR experiments. Capability to measure at the same time all type of charged and neutral particles which can be signature of $\chi$ annihilation would clearly be an experimental advantage: starting from 2006, the AMS-02 experiment on the ISS 


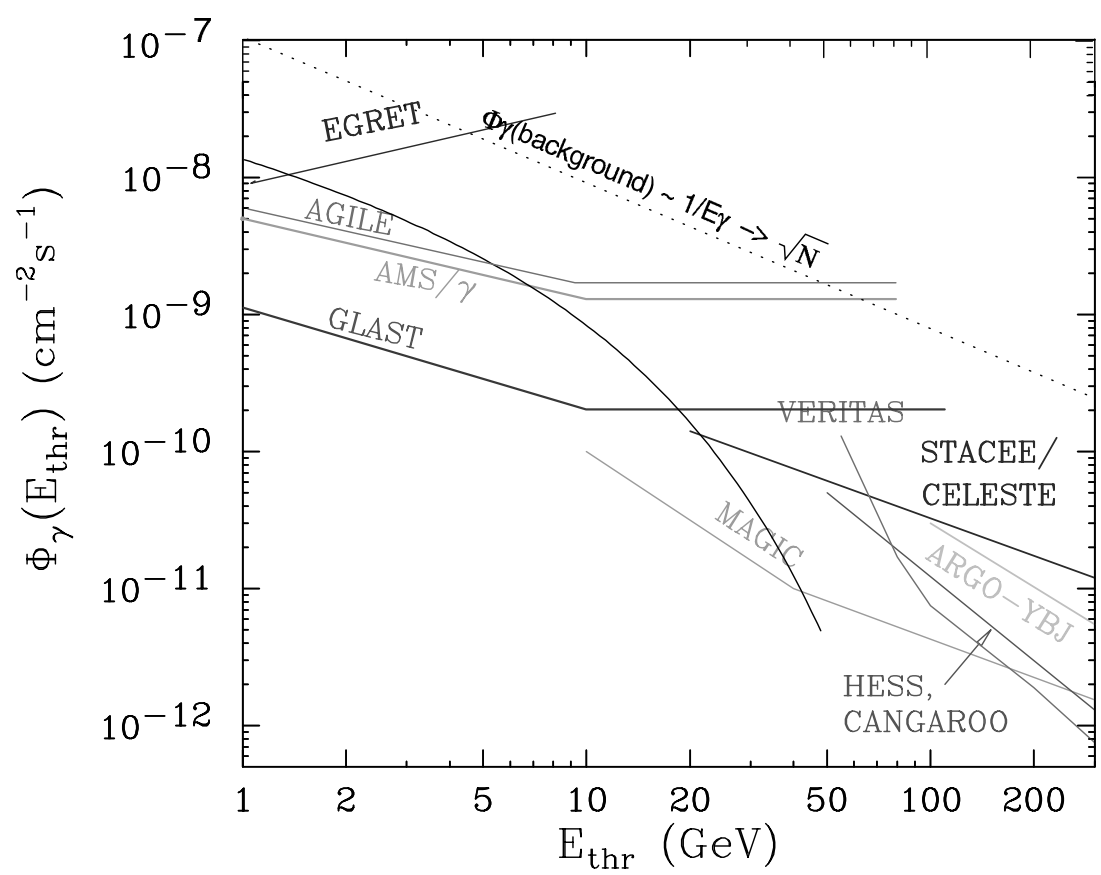

Fig. 10. Sensitivity to SUSY dark matter for various HE gamma ray experiment, either ground based (bottom right) or space based (top left). Vertical axis: gamma rays integral flux above a the energy threshold $E_{t h r}$. The dotted line represent the integral flux of HE gamma rays from known sources, which represent a source of background for this measurement. The continuous line represent the prediction for a SUSY model, with $m_{\chi}=120 \mathrm{GeV}$ and a boost factor $\langle J(0)\rangle=5000[41]$.

will be the only experiment able to precisely measure at the same time all kind of particles related to $\chi$ decay. Other experiment will also contribute to this search: PAMELA will contribute by precisely measure the $\bar{p}$ component, in particular at low energy, starting in 2003, while GLAST will perform the most precise measurement of the high energy $\gamma$ ray halo structure and spectra starting in 2007.

\subsection{Indirect search for ultraheavy particles}

The existence of a measurable flux of Extreme Energies Cosmic Rays above $10^{19} \mathrm{eV}$, represents a big puzzle in modern Astro Particle physics. The behavior of the GKS cutoff versus energy and particle type shown in Figure 7 suggests that no particle except the $\nu$ 's can travel for large distances at these energies. Above $10^{19} \mathrm{eV}$ the volume of the region which can be traversed by hadronic EECR is then dramatically reduced.

One would then expect a steeper spectrum above the GZK cutoff. The experimental results suggest instead a smoother spectrum which would not be possible 

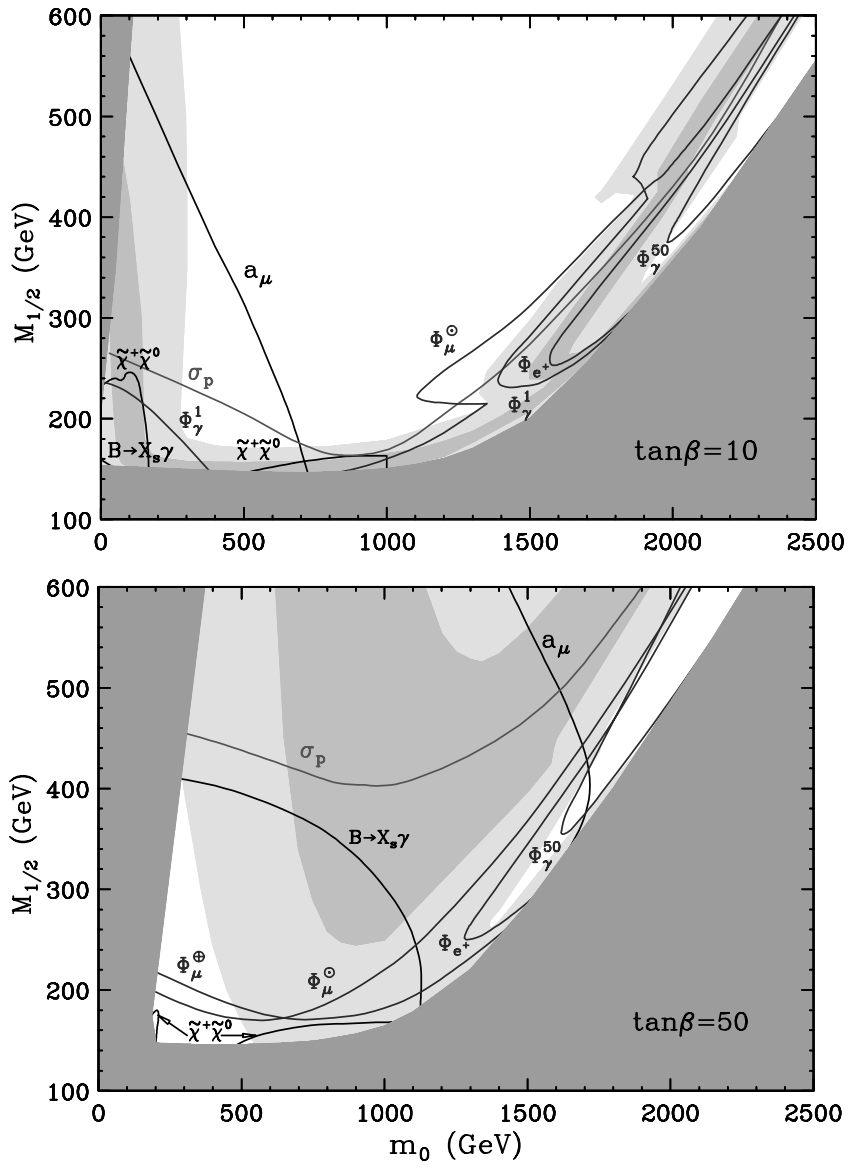

Fig. 11. SUSY parameter space explored by passive experiments. Before the advent of LHC most of the cosmologically interesting parameter space will be explored by ground based or space born experiment. Fore more details see [41]

to explain using conventional physics. Although we are talking only about few dozen events collected by various experiments over several years, their existence challenge standard explanations. One possible scenario is based on EE $\nu$ emitted in the decay of extremely heavy particles present in the very early phases of the universe. These particle travel until they reach regions close to us where they do interact with ordinary IS matter and produce EECR which in turn reach our planet where they can be detected. During this decade the advent of space experiments like EUSO[22] and KLYPVE[23] will improve by one or two orders of magnitude the sensitivity to EECR of the Auger experiment, hopefully clarifying the present situation. 


\section{Conclusion}

One hundred years after their discovery Cosmic Rays have still an important potential for new physics. In order to exploit this potential new, more sensitive experiments are planned which can take advantage of the unique conditions of space for precision measurement of the primary CR flux. During the current decade these experiments might well deliver exciting surprises in Astro Particle physics, on issues like antimatter, dark matter or other exotic states of matter.

\section{Acknowledgments}

This work has been partially supported by the Italian Space Agency (ASI) under contract ARS-98/47.

\section{References}

1. V. Choutko, G. Lamanna, A. Malinin: Int.J. Mod. Phys. A17, 1817 (2002).

2. T. Stanev, Invited talk a the $I I^{\text {nd }}$ International Workshop on Matter, Antimatter and Dark Matter,October 29-30 (2001), unpublished.

3. Y. Ajima, et al. Nucl. Instr. and Methods A443, 71 (2000).

4. R. Bellotti, et al., Phys. Rev. D60, p. 052002, (1999).

5. M. Boezio, et al., ApJ 518, p. 457 (1999).

6. J. W. Mitchell, et al., Proc. $23^{\text {rd }}$ ICRC, 1, 519 (1993).

7. AMS Collaboration, Phys. Rep. 366 (2002).

8. W.R.Webber, et al., Ap.J. 275391 (1983).

9. W.R.Webber, et al., Ap.J. 380 230, (1991).

10. J. Z. Wang, et al., Ap.J. 564244 (2002).

11. E.A. Bogomolov, et al., Proc. $23^{\text {rd }}$ ICRC, 2, 598 (1995).

12. G. Lamanna, et al., Proc. $27^{t h}$ ICRC, 1614 (2001).

13. T.Maeno, et al., Ap. Phys. 16(2) 121 (2001).

14. B. Bertucci, Int.J. Mod. Phys. A17, 1613 (2002).

15. E. Diehl Phys. Rev. D52, 4223 (1995).

16. V. Bonvicini, et al., N.I.M. A461, 262 (2001).

17. R. Battiston, Frascati Physics Series, Vol XXIV, 261 (2002).

18. J. Casaus, Int.J. Mod. Phys. A17, 1603 (2002).

19. T. Sanuki, Int.J. Mod. Phys. A17, 1635 (2002).

20. O. Ganel, et al., AIP Conference Proceedings 458, 272 (1999).

21. J. W. Cronin, AIP Conference Proceedings Volume 566, 1 (2001).

22. L. Scarsi AIP Conference Proceedings Volume 566, 113 (2001).

23. B. A. Khrenov, et al., AIP Conference Proceedings Volume 566, 57 (2001).

24. M. Tavani, Int.J. Mod. Phys. A17, 1799 (2002).

25. R. Battiston, et al., Astropart.Phys. 13, 51 (2000).

26. A. Morselli, Int.J. Mod. Phys. A17, 1829 (2002).

27. K. Greisen, Phys. Rev. Lett. 16, 748 (1966). G.T.Zatsepin, V.A.Kuzmin, JETP Lett. 4, 78 (1966).

28. F. Halzen, this conference.

29. A. Letessier-Selvon, astro-ph/0006111, (2000). 
30. AMS Collaboration, Phys. Lett. B461, 387 (1999).

31. G. Steigmann, Ann. Rev. Astron. Astroph., 14, 339 (1976).

32. E. W. Kolb, M. S. Turner, Ann. Rev. Nucl. Part. Sci. 33, 645 (1983).

33. P. J. E. Peebles, Principles of Physical Cosmology, Princeton University Press, Princeton N.J. (1993).

34. E. Witten, Phys. Rev. D 30, 272 (1984). J. Madsen, astro-ph/9809032, (1998). M. Alford, Ann. Rev. Nucl. Part. Sci. 30131 (2001). K. Rajagopal, F. Wilczek, hep-ph/0011333 (2000).

35. J. Madsen, astro-ph/0112153, (2001).

36. A. Masiero, S. Pascoli, Int.J. Mod. Phys. A17, 1723 (2002).

37. J. Ellis, et al., Phys. Lett. B214, 403, (1988).

38. M.S. Turner, F. Wilzek, Phys. Rev. D 42, 1001 (1990).

39. J. Ellis, astro-ph/9911440, (1999).

40. P. de Bernardis, et al., Frascati Physics Series, Vol XXIV, 399 (2002).

41. J. L. Feng, K. T. Matchev, F. Wilczek, astro-ph/0008115 (2000).

42. F. Donato, P. Fornengo, P. Salati, Phys. Rev. D 62, 043003 (2000).

43. L. Bergström, P. Ullio, J.H. Buckley, astro-ph/9712318, (1997). 